\title{
Liu process and uncertain calculus
}

\author{
Xiaowei Chen ${ }^{1}$ and Dan A Ralescu ${ }^{2 *}$
}

\section{${ }^{*}$ Correspondence:}

ralescd@ucmail.uc.edu

${ }^{2}$ Department of Mathematical

Sciences, University of Cincinnati,

Cincinnati, OH 45221-0025, USA

Full list of author information is

available at the end of the article

\begin{abstract}
Uncertain calculus is a branch of mathematics that deals with the integral and differential of functions of uncertain processes. This paper first introduces the Liu process as an uncertain process defined by the Liu integral. Some properties of Liu processes are investigated such as sample continuity property, finite variation property, and the fact that a continuously differentiable function of the Liu process is another Liu process, among others. Based on the Liu process, the uncertain integral is extended. Furthermore, some mathematical properties are proved, including the fundamental theorem, change of variable theorem, and integration by parts theorem. Finally, uncertain calculus with respect to multiple Liu processes is discussed.
\end{abstract}

Keywords: Uncertainty theory; Uncertain process; Uncertain integral

\section{Introduction}

A stochastic process, a sequence of random variables indexed by time, is a useful tool to deal with dynamical random phenomena. A very important such process is called the Wiener process and was defined by Wiener [1]. The Wiener process, also known as the Brownian motion, is a stochastic process with stationary independent increments, and the increments are normal random variables. Based on the Wiener process, stochastic calculus was developed by Ito [2]. It is a branch of mathematical theory dealing with the integration and differentiation of functions of a stochastic process. Stochastic calculus with respect to the Wiener process is also called the Ito calculus. It has important applications in asset pricing theory. In 1967, the stochastic integral was extended by Kunita and Watanabe [3] to square integrable martingales. Furthermore, stochastic integral with respect to a semimartingale was introduced by Doléans-Dade and Meyer [4]. Besides, stochastic calculus with respect to a local martingale, the Poisson process, and the Lévy process have been studied (see [5,6]).

Stochastic processes are defined based on probability theory. When we use it, a large sample size is needed to estimate probability distribution based on long-run frequency. However, Liu [7] pointed out that the sample size is often too small (even no sample) in practice and the degree of belief usually has much larger variance than the long-run frequency. Thus, we should deal with it by using uncertainty theory. These facts promoted Liu [8] to found an uncertainty theory. That is a branch of mathematics dealing with human uncertainty. In order to describe dynamic uncertain systems, an uncertain process 
was introduced by Liu [9] as a sequence of uncertain variables indexed by time. In addition, Zhang et al. [10] proposed a delayed renewal process. Chen [11] investigated some properties of uncertain stationary independent increments.

Based on the Liu canonical process, a type of uncertain process with stationary independent increments which follow normal uncertainty distribution, Liu [12] invented an uncertain calculus in 2009. This type of uncertain integral is called the Liu integral. It was extended to multiple Liu canonical processes [13]. It is used to deal with the integration and differentiation of uncertain processes. The theory of integration and differentiation of uncertain processes with respect to the Liu process is called the Liu calculus. In order to study an uncertain integral with respect to an uncertain process admitting jumps, an uncertain integral with respect to a renewal process was introduced by Yao [14]. We call this type of uncertain integral the Yao integral. The theory of integration and differentiation of uncertain processes with respect to a renewal process is called the Yao calculus. Chen [15] introduced an uncertain integral with respect to a finite variation process.

In addition, the uncertain differential equation driven by the Liu canonical process was introduced by Liu [9]. After that, Chen and Liu [16] proved the existence and uniqueness theorem for uncertain differential equations. Yao and Gao [17] studied stability theorems for uncertain differential equations. Chen and Liu [16] proposed solution methods for linear uncertain differential equations. Liu [18] and Yao [19] gave a method to solve nonlinear uncertain differential equations. Besides, Yao and Chen [20] introduced a numerical method for uncertain differential equations. Meanwhile, an uncertain differential equation has been applied to uncertain optimal control by Zhu [21], American option pricing by Chen [22], and other option pricing models by Peng and Yao [23]. Liu [24] discussed some basic concepts of uncertain finance. For the latest development of uncertainty theory, please see [25].

In this paper, we generalize the Liu process by the Liu integral. Our goal is to extend an uncertain integral with respect to the Liu process. This uncertain integral has the properties of continuity and linearity. In the framework of the uncertain integral, the fundamental theorem of differentiation of function of uncertain processes is proved. In addition, the integration by parts formula and the formula for change of variables are derived. The rest of the paper is organized as follows: some preliminary concepts of the uncertain process including the Liu calculus, Yao calculus, and uncertain calculus with respect to an uncertain finite variation process are recalled in the 'Preliminary' section. The uncertain integral with respect to the general Liu process will be defined in the 'Liu process' section. The uncertain differential will be discussed in the 'Uncertain integral with respect to the Liu process' section. At last, a brief summary is given in the 'Multifactor Liu process' section.

\section{Preliminary}

The uncertain measure $\mathcal{M}$ is a real-valued set function on a $\sigma$-algebra $\mathcal{L}$ over a nonempty set $\Gamma$ satisfying normality, duality, subadditivity, and product axioms. The triplet $(\Gamma, \mathcal{L}, \mathcal{M})$ is called an uncertainty space.

Definition 1. ([8]) An uncertain variable is a measurable function from an uncertainty space $(\Gamma, \mathcal{L}, \mathcal{M})$ to the set of real numbers, i.e., for any Borel set $\mathrm{B}$ of real numbers, the set

$$
\{\xi \in B\}=\{\gamma \in \Gamma \mid \xi(\gamma) \in B\}
$$

is an event. 
The uncertainty distribution function $\Phi: \Re \rightarrow[0,1]$ of an uncertain variable $\xi$ is defined as $\Phi(x)=\mathcal{M}\{\xi \leq x\}$. The expected value of an uncertain variable is defined as follows.

Definition 2. ([8]) Let $\xi$ be an uncertain variable. Then the expected value of $\xi$ is defined by

$$
E[\xi]=\int_{0}^{+\infty} \mathcal{M}\{\xi \geq r\} \mathrm{d} r-\int_{-\infty}^{0} \mathcal{M}\{\xi \leq r\} \mathrm{d} r
$$

provided that at least one of the two integrals is finite.

The expected value can also be written as

$$
E[\xi]=\int_{0}^{+\infty}(1-\Phi(r)) \mathrm{d} r-\int_{-\infty}^{0} \Phi(r) \mathrm{d} r
$$

where $\Phi(r)$ is the uncertainty distribution of $\xi$. If $\xi$ is an uncertain variable with finite expected value $e$, then the variance of $\xi$ is defined as $\operatorname{Var}[\xi]=E\left[(\xi-e)^{2}\right]$.

Let $\xi_{1}, \xi_{2}, \cdots, \xi_{n}$ be independent uncertain variables with uncertainty distributions $\Phi_{1}, \Phi_{2}, \cdots, \Phi_{n}$, respectively. Liu [26] showed that if the function $f\left(x_{1}, x_{2}, \cdots, x_{n}\right)$ is strictly increasing with respect to $x_{1}, x_{2}, \cdots, x_{m}$ and strictly decreasing with respect to $x_{m+1}, x_{m+2}, \cdots, x_{n}$, then

$$
\xi=f\left(\xi_{1}, \xi_{2}, \cdots, \xi_{n}\right)
$$

is an uncertain variable with inverse uncertainty distribution

$$
\Psi^{-1}(\alpha)=f\left(\Phi_{1}^{-1}(\alpha), \cdots, \Phi_{m}^{-1}(\alpha), \Phi_{m+1}^{-1}(1-\alpha), \cdots, \Phi_{n}^{-1}(1-\alpha)\right) .
$$

Furthermore, Liu and Ha [27] proved that it has an expected value

$$
E[\xi]=\int_{0}^{1} f\left(\Phi_{1}^{-1}(\alpha), \cdots, \Phi_{m}^{-1}(\alpha), \Phi_{m+1}^{-1}(1-\alpha), \cdots, \Phi_{n}^{-1}(1-\alpha)\right) \mathrm{d} \alpha .
$$

Definition 3. ([9]) Let $T$ be an index set and let $(\Gamma, \mathcal{L}, \mathcal{M})$ be an uncertainty space. An uncertain process is a measurable function from $T \times(\Gamma, \mathcal{L}, \mathcal{M})$ to the set of real numbers, i.e., for each $t \in T$ and any Borel set $B$ of real numbers, the set

$$
\left\{X_{t} \in B\right\}=\left\{\gamma \in \Gamma \mid X_{t}(\gamma) \in B\right\}
$$

is an event.

Definition 4. ([9]) Let $\xi_{1}, \xi_{2}, \cdots$ be iid positive uncertain variables. Define

$$
S_{0}=0 \text { and } S_{n}=\xi_{1}+\xi_{2}+\cdots+\xi_{n}
$$

for $n \geq 1$. Then the uncertain process

$$
N_{t}=\max _{n \geq 0}\left\{n \mid S_{n} \leq t\right\}
$$

is called a renewal process.

An uncertain process $X_{t}$ is said to have independent increments if

$$
X_{t_{0}}, X_{t_{1}}-X_{t_{0}}, X_{t_{2}}-X_{t_{1}}, \cdots, X_{t_{k}}-X_{t_{k-1}}
$$


are independent uncertain variables where $t_{0}$ is the initial time and $t_{1}, t_{2}, \cdots, t_{k}$ are any times with $t_{0}<t_{1}<\cdots<t_{k}$. An uncertain process $X_{t}$ is said to have stationary increments if, for any given $t>0$, the increments $X_{s+t}-X_{s}$ are identically distributed uncertain variables for all $s>0$. An uncertain process $S_{t}$ is said to be a stationary independent increment process if it has stationary and independent increments. Liu [26] proved that the expected value of stationary independent increment process $S_{t}$ is $E\left[S_{t}\right]=a+b t$.

Definition 5. ([12]) An uncertain process $C_{t}$ is said to be a canonical Liu process if:

1. $C_{0}=0$ and almost all sample paths are Lipschitz continuous.

2. $C_{t}$ has stationary and independent increments.

3. Every increment $C_{s+t}-C_{s}$ is a normal uncertain variable with expected value 0 and variance $t^{2}$, whose uncertainty distribution is

$$
\Phi(x)=\left(1+\exp \left(\frac{-\pi x}{\sqrt{3} t}\right)\right)^{-1}, x \in \Re .
$$

\section{Liu integral}

In order to deal with the integration and differentiation of uncertain processes, Liu [12] proposed an uncertain integral with respect to the Liu process.

Definition 6. ([12]) Let $X_{t}$ be an uncertain process and $C_{t}$ be a canonical Liu process. For any partition of closed interval $[a, b]$ with $a=t_{1}<t_{2}<\cdots<t_{k+1}=b$, the mesh is written as

$$
\Delta=\max _{1 \leq i \leq k}\left|t_{i+1}-t_{i}\right|
$$

Then the Liu integral of $X_{t}$ is defined as

$$
\int_{a}^{b} X_{t} \mathrm{~d} C_{t}=\lim _{\Delta \rightarrow 0} \sum_{i=1}^{k} X_{t_{i}} \cdot\left(C_{t_{i+1}}-C_{t_{i}}\right)
$$

provided that the limit exists almost surely and is finite. In this case, the uncertain process $X_{t}$ is said to be Liu integrable.

Liu [12] verified the fundamental theorem of uncertain calculus, i.e., for a canonical Liu process $C_{t}$ and a continuously differentiable function $h(t, c)$, the uncertain process $Z_{t}=h\left(t, C_{t}\right)$ has a differential

$$
\mathrm{d} Z_{t}=\frac{\partial h}{\partial t}\left(t, C_{t}\right) \mathrm{d} t+\frac{\partial h}{\partial c}\left(t, C_{t}\right) \mathrm{d} C_{t} .
$$

\section{Yao integral}

In order to study an uncertain integral with respect to an uncertain process admitting jumps, an uncertain integral with respect to a renewal process was introduced by Yao [14].

Definition 7. ([14]) Let $X_{t}$ be an uncertain process and $N_{t}$ be an uncertain renewal process. For any partition of closed interval $[a, b]$ with $a=t_{1}<t_{2}<\cdots<t_{k+1}=b$, the mesh is written as

$$
\Delta=\max _{1 \leq i \leq k}\left|t_{i+1}-t_{i}\right|
$$


Then the Yao integral of $X_{t}$ is defined as

$$
\int_{a}^{b} X_{t} \mathrm{~d} N_{t}=\lim _{\Delta \rightarrow 0} \sum_{i=1}^{k} X_{t_{i}} \cdot\left(N_{t_{i+1}}-N_{t_{i}}\right)
$$

provided that the limit exists almost surely and is finite. In this case, the uncertain process $X_{t}$ is said to be Yao integrable.

Yao [14] verified the fundamental theorem of uncertain calculus, i.e., for a renewal process $N_{t}$ and a continuously differentiable function $h(t, n)$, the uncertain process $Z_{t}=$ $h\left(t, N_{t}\right)$ has a differential

$$
\mathrm{d} Z_{t}=\frac{\partial h}{\partial t}\left(t, N_{t}\right) \mathrm{d} t+h\left(t, N_{t}\right)-h\left(t, N_{t^{-}}\right) .
$$

\section{Uncertain integral with finite variation processes}

Definition 8. ([15]) Let $X_{t}$ be an uncertain process and let $A_{t}$ be a finite variation process. For any partition of closed interval $[a, b]$ with $a=t_{1}<t_{2}<\cdots<t_{k+1}=b$, the mesh is written as

$$
\Delta=\max _{1 \leq i \leq k}\left|t_{i+1}-t_{i}\right| .
$$

Then the uncertain integral of $X_{t}$ with respect to $A_{t}$ is

$$
\int_{a}^{b} X_{t} \mathrm{~d} A_{t}=\lim _{\Delta \rightarrow 0} \sum_{i=1}^{k} X_{t_{i}} \cdot\left(A_{t_{i+1}}-A_{t_{i}}\right)
$$

provided that the limit exists almost surely and is finite. In this case, the uncertain process $X_{t}$ is said to be integrable with respect to the finite variation process $A_{t}$.

Suppose that $A_{t}$ is a finite variation process and $h(t, s)$ a continuously differentiable function, the uncertain process $Z_{t}=h\left(t, A_{t}\right)$ has a differential

$$
\begin{aligned}
\mathrm{d} h\left(t, A_{t}\right)= & \frac{\partial h}{\partial t}\left(t, A_{t}\right) \mathrm{d} t+\frac{\partial h}{\partial x}\left(t, A_{t^{-}}\right) \mathrm{d} A_{t} \\
& -\frac{\partial h}{\partial x}\left(t, A_{t^{-}}\right)\left(A_{t}-A_{t}\right)+h\left(t, A_{t}\right)-h\left(t, A_{t^{-}}\right) .
\end{aligned}
$$

\section{Uncertain differential equation}

Definition 9. ([9]) Suppose $C_{t}$ is a canonical Liu process, and $f$ and $g$ are some given functions. Given an initial value $X_{0}$, then

$$
\mathrm{d} X_{t}=f\left(t, X_{t}\right) \mathrm{d} t+g\left(t, X_{t}\right) \mathrm{d} C_{t}
$$

is called an uncertain differential equation with an initial value $X_{0}$. A solution is an uncertain process $X_{t}$ that satisfies (3) identically in $t$.

Theorem 1. (Existence and Uniqueness Theorem [16]) The uncertain differential equation (3) has a unique solution if the coefficients $f(x, t)$ and $g(x, t)$ satisfy the Lipschitz condition

$$
|f(x, t)-f(y, t)|+|g(x, t)-g(y, t)| \leq L|x-y|, \forall x, y \in \Re, t \geq 0
$$

and linear growth condition 


$$
|f(x, t)|+|g(x, t)| \leq L(1+|x|), \forall x \in \Re, t \geq 0
$$

for some constant L. Moreover, the solution is sample-continuous.

Definition 10. ([20]) The $\alpha$-path $(0<\alpha<1)$ of an uncertain differential equation

$$
\mathrm{d} X_{t}=f\left(t, X_{t}\right) \mathrm{d} t+g\left(t, X_{t}\right) \mathrm{d} C_{t}
$$

with initial value $X_{0}$ is a deterministic function $X_{t}^{\alpha}$ with respect to $t$ that solves the corresponding ordinary differential equation

$$
\mathrm{d} X_{t}^{\alpha}=f\left(t, X_{t}^{\alpha}\right) \mathrm{d} t+\left|g\left(t, X_{t}^{\alpha}\right)\right| \Phi^{-1}(\alpha) \mathrm{d} t
$$

where $\Phi^{-1}(\alpha)$ is the inverse uncertainty distribution of the standard normal uncertain variable, i.e.,

$$
\Phi^{-1}(\alpha)=\frac{\sqrt{3}}{\pi} \ln \frac{\alpha}{1-\alpha}, \quad 0<\alpha<1 .
$$

Theorem 2. (Yao-Chen Formula [20]) Let $X_{t}$ and $X_{t}^{\alpha}$ be the solution and $\alpha$-path of the uncertain differential equation

$$
\mathrm{d} X_{t}=f\left(t, X_{t}\right) \mathrm{d} t+g\left(t, X_{t}\right) \mathrm{d} C_{t},
$$

respectively. Then

$$
\begin{aligned}
& \mathcal{M}\left\{X_{t} \leq X_{t}^{\alpha}, \forall t\right\}=\alpha, \\
& \mathcal{M}\left\{X_{t}>X_{t}^{\alpha}, \forall t\right\}=1-\alpha .
\end{aligned}
$$

The Yao-Chen formula relates an uncertain differential equation and a family of ordinary differential equations just like the Feyman-Kac formula relates a stochastic differential equation and a partial differential equation. Besides, Yao [28] studied the integral of solution to uncertain differential equations.

\section{Liu process}

Definition 11. Let $C_{t}$ be a canonical Liu process, and $\mu_{s}$ and $\sigma_{s}$ be two uncertain processes. Then the uncertain process

$$
X_{t}=X_{0}+\int_{0}^{t} \mu_{s} \mathrm{~d} s+\int_{0}^{t} \sigma_{s} \mathrm{~d} C_{s}
$$

is called a Liu process with drift $\mu_{t}$ and diffusion $\sigma_{t}$. A Liu process $X_{t}$ may also be written in differential form:

$$
\mathrm{d} X_{t}=\mu_{t} \mathrm{~d} t+\sigma_{t} \mathrm{~d} C_{t} .
$$

Example 1. A canonical Liu process $C_{t}$ is a Liu process with initial value 0 , drift 0 , and diffusion 1.

Example 2. An arithmetic Liu process $X_{t}=e t+\sigma C_{t}$ is a Liu process with initial value 0 , drift $e$, and diffusion $\sigma$.

Example 3. A geometric Liu process $X_{t}=\exp \left(e t+\sigma C_{t}\right)$ is a Liu process with initial value 1 , drift $e X_{t}$, and diffusion $\sigma X_{t}$. 
Example 4. The uncertain process $X_{t}=t^{2}+C_{t}^{3}$ is a Liu process with initial value 0 , drift $2 t$, and diffusion $3 C_{t}^{2}$.

Example 5. The solution to an uncertain differential equation

$$
\mathrm{d} X_{t}=f\left(t, X_{t}\right) \mathrm{d} t+g\left(t, X_{t}\right) \mathrm{d} C_{t}
$$

is a Liu process with drift $f\left(t, X_{t}\right)$ and diffusion $g\left(t, X_{t}\right)$.

Example 6. Let $h(t, c)$ be a continuously differentiable function. Then $h\left(t, C_{t}\right)$ is a Liu process with drift $\frac{\partial h}{\partial t}\left(t, C_{t}\right)$ and diffusion $\frac{\partial h}{\partial c}\left(t, C_{t}\right)$.

Next, we will discuss the properties of the path for the Liu process. Let $\Pi: a_{0}=t_{0}<$ $t_{1}<\cdots<t_{n}=t$ be a partition of the closed interval $[0, t]$, and the mesh size of the partition $\Pi$ is defined as $\|\Pi\|_{t}=\max _{1 \leq i \leq n}\left|t_{i}-t_{i-1}\right|$. The total variation of the uncertain process $X_{s}$ over the partition $\Pi$ is defined as

$$
V_{t}[\Pi]=\sum_{i=1}^{n}\left|X_{t_{i}}-X_{t_{i-1}}\right|
$$

Theorem 3. Suppose that $X_{t}$ is a Liu process. Let $\left\{\Pi_{m}\right\}_{m=0}^{\infty}$ be any sequence of partitions of closed interval $[0, t]$ with the mesh size $\lim _{m \rightarrow+\infty}\left\|\Pi_{m}\right\|_{t}=0$. Then the total variation of $X_{t}$ satisfies

$$
\mathcal{M}\left\{\limsup _{m \rightarrow \infty} \sum_{i=1}^{n}\left|X_{t_{i+1}}-X_{t_{i}}\right|=+\infty\right\}=0 .
$$

Proof. Note that

$$
\begin{aligned}
\mathcal{M}\left\{\limsup _{m \rightarrow \infty} V_{t}\left[\Pi_{m}\right]=\infty\right\}= & \mathcal{M}\left\{\limsup _{m \rightarrow \infty} \sum_{i=1}^{n}\left|\int_{t_{i}}^{t_{i+1}} \mu_{s} \mathrm{~d} s+\int_{t_{i}}^{t_{i+1}} \sigma_{s} \mathrm{~d} C_{s}\right|=\infty\right\} \\
\leq & \mathcal{M}\left\{\gamma\left|\limsup _{m \rightarrow \infty} \sum_{i=1}^{n} \int_{t_{i}}^{t_{i+1}}\right| \mu_{s}(\gamma) \mid \mathrm{d} s=\infty\right\} \\
& +\mathcal{M}\left\{\gamma\left|\limsup _{m \rightarrow \infty} \sum_{i=1}^{n} \int_{t_{i}}^{t_{i+1}}\right| \sigma_{s}(\gamma)|\mathrm{d}| C_{s}(\gamma) \mid=\infty\right\} \\
= & \mathcal{M}\left\{\gamma\left|\int_{0}^{t}\right| \mu_{s}(\gamma) \mid \mathrm{d} s=\infty\right\} \\
& +\mathcal{M}\left\{\gamma\left|\int_{0}^{t}\right| \sigma_{s}(\gamma)|\mathrm{d}| C_{s}(\gamma) \mid=\infty\right\} .
\end{aligned}
$$

By the definition of the Liu integral, we know that

$$
\int_{0}^{t} \mu_{s} \mathrm{~d} s+\int_{0}^{t} \sigma_{s} \mathrm{~d} C_{s}
$$

exists almost surely. Then

$$
\int_{0}^{t}\left|\mu_{s}\right| \mathrm{d} s<\infty \text { and } \int_{0}^{t}\left|\sigma_{s}\right| \mathrm{d}\left|C_{s}\right|<\infty \text {, almost surely. }
$$


Thus, we get

$$
\mathcal{M}\left\{\limsup _{\triangle \rightarrow 0} \sum_{i=1}^{k}\left|X_{t_{i+1}}-X_{t_{i}}\right|=+\infty\right\}=0 .
$$

The theorem is proved. Therefore, a Liu process is an uncertain finite variation process.

Theorem 4. The Liu process is a sample-continuous uncertain process.

Proof. In order to prove this theorem, we only need to prove

$$
X_{t+\Delta t}-X_{t}=\int_{t}^{t+\Delta t} \mu_{s} \mathrm{~d} s+\int_{t}^{t+\Delta t} \sigma_{s} \mathrm{~d} C_{s} \rightarrow 0, \text { as } \Delta t \rightarrow 0 .
$$

By the definition of the Liu integral, we know that the integral

$$
\int_{0}^{t} \alpha_{s}(\gamma) \mathrm{d} s+\int_{0}^{t} \sigma_{s}(\gamma) \mathrm{d} C_{s}(\gamma)
$$

is finite for almost all the sample path $\gamma \in \Gamma$. It follows from the continuity of the integral that $\int_{t}^{t+\Delta t} \mu_{s}(\gamma) \mathrm{d} s \rightarrow 0$ and $\int_{t}^{t+\Delta t} \sigma_{s}(\gamma) \mathrm{d} C_{s}(\gamma) \rightarrow 0$ as $\Delta t \rightarrow 0$ almost surely. Therefore, the theorem is proved.

\section{Uncertain integral with respect to the Liu process}

Definition 12. Let $X_{t}$ be a Liu process with drift $\mu_{t}$ and diffusion $\sigma_{t}$, and $Y_{t}$ be an uncertain process. We define an uncertain integral with respect to the Liu process as

$$
\int_{0}^{t} Y_{s} \mathrm{~d} X_{s}=\int_{0}^{t} Y_{s} \mu_{s} \mathrm{~d} s+\int_{0}^{t} Y_{s} \sigma_{s} \mathrm{~d} C_{s}
$$

Theorem 5. The uncertain integral of an uncertain process with respect to the Liu process is also a Liu process.

Proof. For any integrable uncertain process $Y_{t}$ and a Liu process $X_{t}$ with the form

$$
\mathrm{d} X_{t}=\mu_{t} \mathrm{~d} t+\sigma_{t} \mathrm{~d} C_{t},
$$

the uncertain integral $Z_{t}=\int_{0}^{t} Y_{s} \mathrm{~d} X_{s}$ is

$$
Z_{t}=\int_{0}^{t} Y_{s} \mu_{s} \mathrm{~d} s+\int_{0}^{t} Y_{s} \sigma_{s} \mathrm{~d} C_{s} .
$$

It follows from the definition of the Liu process that $Z_{t}$ is indeed a Liu process.

Theorem 6. Suppose that $X_{t}$ is a Liu process and $Y_{t}$ is an integrable uncertain process with respect to $X_{t}$ on interval $[a, b]$. Then it is integrable on each subinterval of $[a, b]$. Moreover, if $c \in[a, b]$, then

$$
\int_{a}^{b} Y_{t} \mathrm{~d} X_{t}=\int_{a}^{c} Y_{t} \mathrm{~d} X_{t}+\int_{c}^{b} Y_{t} \mathrm{~d} X_{t}
$$


Proof. Let $\left[a_{0}, b_{0}\right]$ be a subinterval of $[a, b]$. By the definition of the uncertain integral with respect to the Liu process, we get

$$
\int_{a}^{b} Y_{s} \mathrm{~d} X_{s}=\int_{a}^{b} Y_{s} \mu_{s} \mathrm{~d} s+\int_{a}^{b} Y_{s} \sigma_{s} \mathrm{~d} C_{s}
$$

Since $Y_{s}$ is integrable on $[a, b], Y_{s} \mu_{s}$ and $Y_{s} \sigma_{s}$ are integrable on the interval $[a, b]$ with respect to $t$ and $C_{t}$, respectively. Then $Y_{s} \mu_{s}$ and $Y_{s} \sigma_{s}$ are integrable on any subinterval of $[a, b]$. Therefore, we obtain

$$
\int_{a^{\prime}}^{b^{\prime}} Y_{s} \mathrm{~d} X_{s}=\int_{a^{\prime}}^{b^{\prime}} Y_{s} \mu_{s} \mathrm{~d} s+\int_{a^{\prime}}^{b^{\prime}} Y_{s} \sigma_{s} \mathrm{~d} C_{s}
$$

Next,

$$
\begin{aligned}
\int_{a}^{b} Y_{s} \mathrm{~d} X_{s} & =\int_{a}^{b} Y_{s} \mu_{s} \mathrm{~d} s+\int_{a}^{b} Y_{s} \sigma_{s} \mathrm{~d} C_{s} \\
& =\int_{a}^{c} Y_{s} \mu_{s} \mathrm{~d} s+\int_{a}^{c} Y_{s} \sigma_{s} \mathrm{~d} C_{s}+\int_{c}^{b} Y_{s} \mu_{s} \mathrm{~d} s+\int_{c}^{b} Y_{s} \sigma_{s} \mathrm{~d} C_{s} \\
& =\int_{a}^{c} Y_{s} \mathrm{~d} X_{s}+\int_{c}^{b} Y_{s} \mathrm{~d} X_{s} .
\end{aligned}
$$

Hence, the theorem is proved.

Theorem 7. Suppose that $X_{t}$ is a Liu process, $Y_{t}$ and $Z_{t}$ are integrable uncertain processes with respect to the Liu process $X_{t}$ on $[a, b]$, and $\alpha$ and $\beta$ are real numbers. Then

$$
\int_{a}^{b}\left(\alpha Y_{t}+\beta Z_{t}\right) \mathrm{d} X_{t}=\alpha \int_{a}^{b} Y_{t} \mathrm{~d} X_{t}+\beta \int_{a}^{b} Z_{t} \mathrm{~d} X_{t}
$$

Proof. It follows from the definition of the uncertain integral with respect to the Liu process that

$$
\begin{aligned}
\int_{a}^{b}\left(\alpha_{t} Y_{t}+\beta Z_{t}\right) \mathrm{d} X_{t}= & \int_{a}^{b}\left(\alpha_{t} Y_{t}+\beta Z_{t}\right) \mu_{t} \mathrm{~d} t+\int_{a}^{b}\left(\alpha_{t} Y_{t}+\beta Z_{t}\right) \sigma_{t} \mathrm{~d} C_{t} \\
= & \int_{a}^{b} \alpha_{t} Y_{t} \mu_{t} \mathrm{~d} t+\int_{a}^{b} \beta Z_{t} \mu_{t} \mathrm{~d} t \\
& +\int_{a}^{b} \alpha_{t} Y_{t} \sigma_{t} \mathrm{~d} C_{t}+\int_{a}^{b} \beta Z_{t} \sigma_{t} \mathrm{~d} C_{t} \\
= & \alpha\left(\int_{a}^{b} Y_{t} \mu_{t} \mathrm{~d} t+\int_{a}^{b} Y_{t} \sigma_{t} \mathrm{~d} C_{t}\right) \\
& +\beta\left(\int_{a}^{b} Z_{t} \mu_{t} \mathrm{~d} t+\int_{a}^{b} Z_{t} \sigma_{t} \mathrm{~d} C_{t}\right) \\
= & \alpha \int_{a}^{b} Y_{t} \mathrm{~d} X_{t}+\beta \int_{a}^{b} Z_{t} \mathrm{~d} X_{t} .
\end{aligned}
$$

The theorem is proved. 
Theorem 8. (Fundamental Theorem) Let $X_{t}$ be a Liu process with drift $\mu_{t}$ and diffusion $\sigma_{t}$ and $f(t, x)$ be a continuously differentiable function. Then $f\left(t, X_{t}\right)$ is a Liu process with

$$
\begin{aligned}
\mathrm{d} f\left(t, X_{t}\right) & =\frac{\partial f}{\partial t}\left(t, X_{t}\right) \mathrm{d} t+\frac{\partial f}{\partial x}\left(t, X_{t}\right) \mathrm{d} X_{t} \\
& =\left(\frac{\partial f}{\partial t}\left(t, X_{t}\right)+\frac{\partial f}{\partial x}\left(t, X_{t}\right) \mu_{t}\right) \mathrm{d} t+\frac{\partial f}{\partial x}\left(t, X_{t}\right) \sigma_{t} \mathrm{~d} C_{t} .
\end{aligned}
$$

Proof. Since the function $f$ is continuously differentiable, by using the Taylor series expansion of $f\left(t, X_{t}\right)$ with respect to all its arguments, which in this case are $t$ and $X_{t}$, we take the Taylor series expansion out to the first order of $t$ and $X_{t}$. Then the infinitesimal increment of $f\left(t, X_{t}\right)$ has a first-order approximation

$$
\begin{aligned}
\Delta f\left(t, X_{t}\right) & =\frac{\partial f}{\partial t}\left(t, X_{t}\right) \Delta t+\frac{\partial f}{\partial x}\left(t, X_{t}\right) \Delta X_{t} \\
& =\left(\frac{\partial f}{\partial t}\left(t, X_{t}\right)+\frac{\partial f}{\partial x}\left(t, X_{t}\right) \mu_{t}\right) \Delta t+\frac{\partial f}{\partial x}\left(t, X_{t}\right) \sigma_{t} \Delta C_{t} .
\end{aligned}
$$

In fact, the uncertain differential (8) is equivalent to the uncertain integral equation

$$
f\left(t, X_{t}\right)=f\left(0, X_{0}\right)+\int_{0}^{t} \frac{\partial f}{\partial t}\left(s, X_{s}\right) \mathrm{d} s+\int_{0}^{t} \frac{\partial f}{\partial x}\left(s, X_{s}\right) \mathrm{d} X_{s} .
$$

Theorem 9. (Change of Variables) Let $f$ and $g$ be continuously differentiable functions. Then for any $s>0$, we have

$$
\int_{0}^{t} f^{\prime}\left(g\left(X_{s}\right)\right) g^{\prime}\left(X_{s}\right) \mathrm{d} X_{s}=f\left(g\left(X_{t}\right)\right)-f\left(g\left(X_{0}\right)\right) .
$$

Proof. Since $f$ and $g$ are continuously differentiable functions, it follows from the fundamental theorem that

$$
\mathrm{d} f\left(g\left(X_{t}\right)\right)=f^{\prime}\left(g\left(X_{t}\right)\right) g^{\prime}\left(X_{t}\right) \mathrm{d} X_{t} .
$$

Then we get

$$
f\left(g\left(X_{t}\right)\right)=f\left(g\left(X_{0}\right)\right)+\int_{0}^{t} f^{\prime}\left(g\left(X_{s}\right)\right) g^{\prime}\left(X_{s}\right) \mathrm{d} X_{s} .
$$

Theorem 10. (Integration by Parts) Suppose that $X_{t}$ is a Liu process and $F(x)$ is a continuously differentiable function. Then

$$
\int_{0}^{t} F(s) \mathrm{d} X_{s}=F(t) X_{t}-F(0) X_{0}-\int_{0}^{t} X_{s} \mathrm{~d} F(s) .
$$

Proof. It follows from the fundamental theorem that

$$
\mathrm{d}\left(F(t) X_{t}\right)=F^{\prime}(t) X_{t} \mathrm{~d} t+F(t) \mathrm{d} X_{t} .
$$

Thus,

$$
\begin{aligned}
\int_{0}^{t} F(s) \mathrm{d} X_{s} & =F(t) X_{t}-F(0) X_{0}-\int_{0}^{t} F^{\prime}(s) X_{s} \mathrm{~d} s \\
& =F(t) X_{t}-F(0) X_{0}-\int_{0}^{t} X_{s} \mathrm{~d} F(s)
\end{aligned}
$$




\section{Multifactor Liu process}

Definition 13. Let $C_{1 t}, C_{2 t}, \cdots, C_{n t}$ be canonical Liu processes, and $\mu_{t}$ and $\sigma_{1 t}, \sigma_{2 t}, \cdots$, $\sigma_{n t}$ be uncertain processes. Then the uncertain process

$$
X_{t}=X_{0}+\int_{0}^{t} \mu_{s} \mathrm{~d} s+\sum_{i=1}^{n} \int_{0}^{t} \sigma_{i s} \mathrm{~d} C_{i s}
$$

is called a multifactor Liu process with drift $\mu_{t}$ and diffusions $\sigma_{1 t}, \sigma_{2 t}, \cdots, \sigma_{n t}$. The Liu process $X_{t}$ may also be written in differential form:

$$
\mathrm{d} X_{t}=\mu_{t} \mathrm{~d} t+\sum_{i=1}^{n} \sigma_{i t} \mathrm{~d} C_{i t} .
$$

Definition 14. Let $X_{t}$ be a multifactor Liu process with drift $\mu_{t}$ and diffusions $\sigma_{1 t}, \sigma_{2 t}, \cdots, \sigma_{n t}$ and $Y_{t}$ be an uncertain process. We define an uncertain integral with respect to the multifactor Liu process as

$$
\int_{0}^{t} Y_{s} \mathrm{~d} X_{s}=\int_{0}^{t} Y_{s} \mu_{s} \mathrm{~d} s+\sum_{i=1}^{n} \int_{0}^{t} Y_{s} \sigma_{i s} \mathrm{~d} C_{i s}
$$

Theorem 11. (Multifactor Fundamental Theorem) Let $Y_{1 t}, Y_{2 t}, \cdots, Y_{n t}$ be Liu processes. If $f\left(t, y_{1}, y_{2}, \cdots, y_{n}\right)$ is a continuously differentiable function, then the uncertain process $Z_{t}=h\left(t, Y_{1 t}, Y_{2 t}, \cdots, Y_{n t}\right)$ is a Liu process with

$$
\mathrm{d} Z_{t}=\frac{\partial f}{\partial t}\left(t, Y_{1 t}, Y_{2 t}, \cdots, Y_{n t}\right) \mathrm{d} t+\sum_{i=1}^{n} \frac{\partial f}{\partial y_{i}}\left(t, Y_{1 t}, Y_{2 t}, \cdots, Y_{n t}\right) \mathrm{d} Y_{i t}
$$

Proof. Since the function $f$ is continuously differentiable, by using the Taylor series expansion, the infinitesimal increment of $Z_{t}$ has a first-order approximation

$$
\begin{aligned}
\Delta f\left(t, X_{t}\right)= & \frac{\partial f}{\partial t}\left(t, Y_{1 t}, Y_{2 t}, \cdots, Y_{n t}\right) \Delta t \\
& +\sum_{i=1}^{n} \frac{\partial f}{\partial y_{i}}\left(t, Y_{1 t}, Y_{2 t}, \cdots, Y_{n t}\right) \Delta Y_{i t} .
\end{aligned}
$$

Example 7. Suppose that $X_{t}$ and $Y_{t}$ are two Liu processes. Since

$$
\frac{\partial(x y)}{\partial t}=0, \frac{\partial(x y)}{\partial x}=y, \text { and } \frac{\partial(x y)}{\partial y}=x,
$$

the uncertain process $X_{t} Y_{t}$ is a Liu process with

$$
\mathrm{d} X_{t} Y_{t}=X_{t} \mathrm{~d} Y_{t}+Y_{t} \mathrm{~d} X_{t}
$$

\section{Conclusions}

This paper introduced the concept of the Liu process which is defined by the Liu integral. Based on the Liu process, we extended the Liu integral on such a process. Some basic properties of this integral were discussed. Furthermore, the uncertain differential was introduced, and the fundamental theorem of uncertain calculus was derived. The integration by parts theorem was also discussed. Finally, we have studied multifactor Liu processes. 


\section{Acknowledgements}

This work was supported by the National Natural Science Foundation of China (grant no. 61273044), Nankai University Project Funds for Young Teachers (no. NKQ1118), and Tianjin Municipal Research Program of Application Foundation and Advanced Technology of China (grant no. 10JCYBJC07300).

\section{Author details}

${ }^{1}$ Department of Risk Management and Insurance, Nankai University, Tianjin 300071, China. ${ }^{2}$ Department of Mathematical Sciences, University of Cincinnati, Cincinnati, OH 45221-0025, USA.

Received: 16 February 2013 Accepted: 19 April 2013

Published: 19 June 2013

\section{References}

1. Wiener, N: Differential space. J. Math. Phys. 2, 131-174 (1923)

2. Ito, K: Stochastic integral. Proc. Jpn. Acad. 20, 519-524 (1944)

3. Kunita, H, Watanabe, S: On square integrable martingales. Nagoya Math. 30, 209-245 (1967)

4. Doléans-Dade, C, Meyer, P: Universté de strasbourg séminaire de probabilités. Lect. Notes Math. 5, 143-173 (1970)

5. Jacod, J: Calculus stocastique et problemes de martingales. Lect. Notes Math. 714, 66-112 (1979)

6. Kallenberg, O: Foundations of Modern Probability. 2nd edn. Springer, New York (1997)

7. Liu, B: Why is there a need for uncertainty theory? J. Uncertain Syst. 6(1), 3-10 (2012)

8. Liu, B: Uncertainty Theory. 2nd edn. Springer, Berlin (2007)

9. Liu, B: Fuzzy process, hybrid process and uncertain process. J. Uncertain Syst. 2(1), 3-16 (2008)

10. Zhang, $X$, Ning, $Y$, Meng, G: Delayed renewal process with uncertain interarrival times. Fuzzy Optimization Decis. Mak. 12(1), 79-87 (2013)

11. Chen, $X$ : Variation analysis of uncertain stationary independent increment processes. Eur. J. Oper. Res. 222(2), 312-316 (2012)

12. Liu, B: Some research problems in uncertainty theory. J. Uncertain Syst. 3(1), 3-10 (2009)

13. Liu, B, Yao, K: Uncertain integral with respect to multiple canonical processes. J. Uncertain Syst. 6(4), 250-255 (2012)

14. Yao, K: Uncertain calculus with renewal process. Fuzzy Optimization Decis. Mak. 11(3), 285-297 (2012)

15. Chen, X: Uncertain calculus with respected to finite variation processes. Technical Report. (2013). http://orsc.edu.cn/ online/110303.pdf

16. Chen, $X, L i u, B:$ Existence and uniqueness theorem for uncertain differential equations. Fuzzy Optimization Decis. Mak. $\mathbf{9}(1), 69-81(2010)$

17. Yao, K, Gao, J, Gao, Y: Some stability theorems of uncertain differential equation. Fuzzy Optimization Decis. Mak. 12(1), 3-13 (2013)

18. Liu, Y: An analytic method for solving uncertain differential equations. J. Uncertain Syst. 6(4), 244-249 (2012)

19. Yao, K: A type of nonlinear uncertain differential equations with analytic solution. (2013). http://orsc.edu.cn/online/ 110928.pdf

20. Yao, K, Chen, X: A numerical method for solving uncertain differential equations. J. Intell. Fuzzy Syst (2013). in press

21. Zhu, Y: Uncertain optimal control with application to a portfolio selection model. Cybern. Syst. 41 (7), 535-547 (2010)

22. Chen, $X$ : American option pricing formula for uncertain financial market. Int. J. Oper. Res. 8(2), 32-37 (2011)

23. Peng, J, Yao, K: A new option pricing model for stocks in uncertainty markets. Int. J. Oper. Res. 7(4), 213-224 (2010)

24. Liu, B: Toward uncertain finance theory. J. Uncertainty Anal. Appl. 1, 1 (2013)

25. Liu, B: Uncertainty Theory, 4th edn. (2013). http//:orsc.edu.cn/liu/ut.pdf

26. Liu, B: Uncertainty Theory: A Branch of, Mathematics for Modeling Human Uncertainty. Springer, Berlin (2010)

27. Liu, Y, Ha, M: Expected value of function of uncertain variables. J. Uncertain Syst. 4(3), 181-186 (2010)

28. Yao, K: Extreme values and integral of solution of uncertain differential equation. J. Uncertainty Anal. Appl. 1, 3 (2013)

doi:10.1186/2195-5468-1-3

Cite this article as: Chen and Ralescu: Liu process and uncertain calculus. Journal of Uncertainty Analysis and Applications 2013 1:3.

\section{Submit your manuscript to a SpringerOpen ${ }^{\circ}$ journal and benefit from:}

- Convenient online submission

- Rigorous peer review

- Immediate publication on acceptance

- Open access: articles freely available online

- High visibility within the field

- Retaining the copyright to your article

Submit your next manuscript at $\boldsymbol{\triangleright}$ springeropen.com 